\title{
Association of Increased Levels of Happiness with Reduced Levels of Tension and Anxiety after Mental Stress Testing in Japanese College Students
}

\author{
Satoshi Horiuchi ${ }^{1,2}$, Akira Tsuda ${ }^{3}$, Natsuki Toyoshima ${ }^{4}$, Shuntaro Aoki ${ }^{5}$, Yuji Sakano ${ }^{2}$ \\ ${ }^{1}$ Japan Society for the Promotion of Science, Kojimachi, Japan \\ ${ }^{2}$ School of Psychological Science, Health Sciences University of Hokkaido, Sapporo, Japan \\ ${ }^{3}$ Department of Psychology, Kurume University, Kurume, Japan \\ ${ }^{4}$ Graduate School of Psychology, Kurume University, Kurume, Japan \\ ${ }^{5}$ Graduate School of Psychological Science, Health Sciences University of Hokkaido, Sapporo, Japan \\ Email: satosato.007@nifty.com
}

Received June $3^{\text {rd }}, 2013$; revised July $6^{\text {th }}, 2013$; accepted August $2^{\text {nd }}, 2013$

Copyright (C) 2013 Satoshi Horiuchi et al. This is an open access article distributed under the Creative Commons Attribution License, which permits unrestricted use, distribution, and reproduction in any medium, provided the original work is properly cited.

\begin{abstract}
Previous studies from western countries have reported that happy individuals report lower levels of negative mood during and/or following mental stress testing; this finding has not been examined in Japan. This study examined the relationship between happiness, measured using the Subjective Happiness Scale (Lyubomirsky \& Lepper, 1999), and negative moods (i.e., tension and anxiety) during and after mental stress testing in Japanese college students. Based on the findings of previous literature and inverse correlations between positive and negative moods, we hypothesized that participants with higher levels of happiness (the higher happiness group, or HG) show significantly lower levels of negative moods and higher levels of positive moods following mental stress testing, compared to participants with lower levels of happiness (the lower happiness group, or LG). Of a total of 392 Japanese undergraduates who participated in a screening survey, those whose scores were one standard deviation higher or lower than the average score were invited to participate in the experiment. Eight HG and nine LG students agreed to participate. A five-minute computerized mental arithmetic task was used to induce stress. The session comprised a five minute pre-task period, a five minute task, and a five minute post-task period. The levels of positive and negative moods during each period were measured retrospectively following each period. Heart rate was measured during the session. Participant heart rate levels and negative moods increased significantly from the pre-task to the task periods, and subsequently decreased during the post-task period. Levels of positive mood decreased from the pre-task to the task period. Negative moods were significantly lower in HGs than in LGs during the post-task period. These results partially supported the hypothesis whereby subjective happiness buffered the impact of stressors on negative moods by influencing post-stress negative mood levels.
\end{abstract}

Keywords: Happiness; Mental Stress Testing; Mood; Stress

\section{Introduction}

Stress management is an important issue for maintaining health (Greenberg, 2010). Stress is related not only to increased risks in relation to numerous chronic diseases, such as cardiovascular diseases (McEwen, 1998), but also decreased productivity (Watts \& Robertson, 2011). Despite such negative effects, several individuals experience stress globally, including the United States (Anderson et al., 2010) and Japan (Cabinet Office of Japan, 2008). For example, more than $60 \%$ of Japanese adults aged 20 - 59 experience stress on a daily basis (Cabinet Office of Japan, 2008). While the acquisition of stress management skills is important, increased psychological resources are also effective and can buffer the adverse impacts of stressful situations. Such psychological interventions presuppose the identification of stress-buffering resources.

In recent times, the importance of positive psychological factors has been recognized in stress-related sciences (Nelson \& Cooper, 2005); one such factor is happiness. Happiness has been used to refer to positive subjective experiences (Schiffrin \& Nelson, 2010). For example, happiness has been used to refer to life satisfaction, the presence of a positive affect, the absence of a negative affect, and/or a global, subjective evaluation of whether one is happy (Lyubomirsky \& Lepper, 1999). As individuals, we have the ability to evaluate our happiness (Lyubomirsky \& Lepper, 1999). However, this is highly subjective across individuals on the basis of what an individual deems as being happy; such an evaluation does not necessarily correspond to a simple sum of life satisfaction and affective experiences (Lyubomirsky, Tkach, \& DiMatteo, 2006). Therefore, it is important to focus on global evaluations as well as specific components of happiness such as life satisfaction and positive affect. Therefore, this study focuses on an individual's global and subjective assessment of happiness. 
Based on the transactional model of stress and coping (Lazarus \& Folkman, 1984), this study defines stress as the relationship between the quality of a certain stressful situation (stressor) and an individual's coping resources in relation to the stressor. A common stressor faced by most people is daily hassle (Stawski, Sliwinski, Almeida, \& Smyth, 2008). Daily hassles are minor, but can occur frequently. Lazarus (2009) asserted that daily hassles can exert long-term effects on physical and mental well-being. Generally, it is assumed that a given daily hassle increases negative mood/affect, such as tension or irritation. These reactions are transient in nature; however, the intensity and return to baseline levels varies among individuals. Importantly, the magnitude of mood reactivity (Stawski et al., 2008 ) is related to levels of stress (strain). Greater levels of reactivity result in higher levels of stress. Furthermore, it is suggested that insufficient psychological and physiological recovery is a critical pathway through which stress can exert adverse effects on health (Brosschot, Gerin, \& Thayer, 2006; McEwen, 1998).

Previous literature examined relationships between happiness, a global evaluation of individual happiness, and mood levels during stressful situations. Lyubomirsky and Ross (1997) reported that unhappy students, unlike their happy counterparts, showed decreased positive moods when they completed anagrams with a faster peer. Lyubomirsky, Tucker, and Kasri (2001) reported that happy participants exhibited decreased levels of positive affect compared to unhappy counterparts following the news that their team lost in a word-generation task. Other studies focused on traits associated with positive affect. Bostock, Hamer, Wawrzyniak, Mitchell, and Steptoe (2011) found significant relationships between positive emotional styles (e.g., happy, cheerful, lively) measured using an ecological momentary assessment over a two-day period, and the tension and anxiety during a five-minute mirror-tracing task and a five-minute socially evaluating speech task. In contrast, Steptoe, Gibson, Hamer, and Wardle (2007) reported that levels of happiness measured using ecological momentary assessment over a two-day period was not related to the magnitude of subjective stress response during a five-minute mirror-tracing task and a three-minute public-speech task.

Few studies have examined relationships between trait positive affect and negative moods following mental stress testing, however, results have been inconsistent. Papousek, Nauschnegg, Paechter, Lackner, Goswami, and Schulter (2010) reported that students with higher levels of trait positive affect, measured using Positive Affect Negative Affect Scale (PANAS) (Watson, Clark, \& Tellegen, 1988), tended to show lower levels of subjective tension after answering difficult statistical questions. In contrast, Bostock et al. (2011) found no significant relationships between positive emotional style, tension, and anxiety following mental stress testing.

These results show that higher levels of happiness or trait positive affect is associated with lower levels of negative moods during and after mental stress testing. However, results have been inconsistent across studies. Inconsistent results may be explained by differences in measurements of positive affect or happiness, those in experimental procedures, and those in participants. Because a variety of happiness-measuring methods have been developed (Lyubomirsky, 2000), the improvement of stress buffering effects of happiness with each measure is vital. Little is known whether happiness, which is measured using the Subjective Happiness Scale (SHS) (Lyubomirsky \& Lepper,
1999), is associated with improved moods following mental stress testing. In addition, most previous studies have been based on western societies, such as the United States; however, similar studies based on Japan that examine the relationships between happiness and changes in mood responses over time have not been conducted.

This study examines the relationship between happiness and negative mood during and after mental stress testing in Japanese students. We measure happiness using Lyubomirsky \& Lepper's (1999) SHS. We establish and test the following two hypotheses.

Hypothesis 1: Happier individuals have significantly lower levels of negative and higher levels of positive mood during mental stress testing.

Hypothesis 2: Happier individuals have significantly lower levels of negative and higher levels of positive mood following mental stress testing.

Examining the relationship between happiness and mood responses during and after mental stress testing in Japan provides several theoretical and practical implications. Theoretically, the examination tests the external validity of the findings originating in western countries. The cross-cultural definition of "happiness" may differ particularly between western and eastern cultures. While personal and internal aspects of happiness are emphasized in western countries, social aspects of happiness are emphasized in eastern cultures (Uchida \& Kitayama, 2009). Happiness is strongly related to self-associated factors, such as self-esteem (Campbell, 1981) and personal accomplishment (Emmons, 1986), in North America, while it correlates more strongly with social factors, such as perceived emotional support (Uchida, Kitayama, Mesquita, Reyes, \& Morling, 2008) and relational harmony (Kwan, Bond, \& Singelis, 1997), in East Asian countries. A practical demonstration of the moodbuffering effects of happiness will provide the rationale to health promotion practitioners to incorporate happiness-enhancing interventions in stress management programs in Japan.

\section{Method}

\section{Sample Selection}

Participants for this study were selected from a college located in Fukuoka, Japan. A total of 392 college students completed the Japanese version of the SHS (JSHS; Shimai, Otake, Utsuki, Ikemi, \& Lyubomirsky, 2004), as well as other measures of stress (data not shown). The JSHS has been found to be reliable and valid (Shimai et al., 2004). These participants were requested to provide their e-mail addresses in the case of voluntarily consenting to participate in an additional experimental study. These surveys were conducted during introductory or health psychology classes after obtaining permission from the class lecturer. The average JSHS score was 4.40 , with a standard deviation (SD) of 1.09. This study defined happier students as those whose score was higher than 5.49 (mean + 1SD), while less happy ones as those whose score was lower than 3.38 (mean - 1SD), making it possible to divide the participants into two distinct groups - the higher happiness group (HG) and the lower happiness group (LG). A total of 76 and 60 students qualified as HG and LG participants, respectively. Those students who provided their e-mail addresses and qualified as participants in one of the two groups were invited to participate in the present study. Consequently, eight HG and nine LG students agreed to participate. The participants mean 
age was 21.5 years and a SD of 2.07. Of the 17 participants, six were male. Both groups were similar in age $(\mathrm{t}(15)=0.40$, n.s. $)$ and sex ratio $\left(\chi^{2}(1)=0.40\right.$, n.s. $)$.

\section{Mental Stress Testing}

A mental arithmetic task involved adding pairs of two-digit numbers as promptly and accurately as possible in front of an evaluative observer. The mental arithmetic task was computerized. In each trial, a pair of two-digit numbers (e.g., $64+34$ ) was presented at the center of the screen, with four potential answers (e.g., 98, 108, 88, and 78) at the bottom. Each participant was required to solve each problem and choose the correct answer among the four choices within 10 seconds. A timer was displayed at the bottom of the screen.

\section{Measures}

The Japanese UWIST mood adjective checklist (Okamura, Tsuda, \& Yajima, 2004) comprises 12 items that measure the fundamental dimensions of energetic and tense arousal. Each question was rated on a four-point scale and the scores were totaled so that higher energetic arousal and tension arousal values reflect greater energetic arousal and tension, respectively. Tension arousal has been reported to increase during mental stress testing (Horiuchi, Tsuda, Okamura, Yajima, \& Steptoe, 2010). The Japanese UWIST mood adjective checklist has been found to be reliable and valid (Okamura et al., 2004).

\section{Procedure}

The methodology employed for this study was approved by the ethics committee of the first author's college. After entering the experimental room (a sound-attenuated small chamber, $2 \mathrm{~m}$ $\times 3 \mathrm{~m}$ ), the study's purpose and the experimental procedure were disclosed to all the participants. Each participant provided informed consent.

The session consisted of a five-minute pre-task period, a five-minute task period, and a five-minute post-task period. Each participant was asked to relax during the three tasks in the presence of an observer, as described above. After each period, participants completed the mood check list. The experimenter instructed the participant to evaluate their mood during each period.

Heart rate was continuously measured during the experiment using the Heart Rhythm Scanner 2.0 (Biocom Technologies, San Francisco, CA) and was assessed as an objective indicator of stress. Although an increased heart rate does not necessarily reflect negative emotion or mental workload, it is a useful and objective index of stress. Previous studies (Bostock et al., 2011; Brummett, Boyle, Kuhn, Siegler, \& Williams, 2009; Steptoe et al., 2007) consistently reported that heart rate increases during mental stress testing, independent of happiness or trait positive affect. An exception was a study by Horiuchi, Tsuda, Hashimoto, Kai, and Wenjie (2008). It reported that heart rates were significantly higher in low-happiness participants during the pre-task period and the mental arithmetic task, but not during the speech task and the post-task period. However, they required each participant to complete two tasks (talking about pleasurable experiences in front of a video camera and mental arithmetic), one of which may have induced positive moods. Matsunaga, Murakami, Yamakawa, Isowa, Fukuyama, Shinoda, et al. (2011) reported that positive emotions were more strongly evoked in happier individuals than less happy individuals when a favorite person was presented as stimuli. Thus, the validity of these tasks as mental stress tests is questionable.

\section{Statistical Analyses}

Prior to the analyses of mood responses, validity of mental stress testing was examined. It was predicted that heart rate would significantly increase from the pre-task to the task period, and subsequently decrease during the post-task period. Performance and subjective demand ratings were also compared. Finally, patterns of mood responses were compared between groups. The significance level was set below $5 \%$.

\section{Results}

\section{Performance and Heart Rate Responses}

The number of the total trials was $105.5 \pm 4.91$ on an average and correct trials had a mean of $78.0 \pm 8.22$. The percentage of the correct trials was $74.0 \pm 7.27$ on an average. A series of unpaired t-tests revealed that there were no significant differences in the number of total trials $(\mathrm{t}(15)=0.41$, n.s.), correct answers $(\mathrm{t}(15)=2.08$, n.s.), and percentage of correct answers $(\mathrm{t}(15)=2.06$, n.s.). Figure 1 shows heart rate responses during each period. Heart rate averaged $80.0 \pm 8.61$ beats per minute (BPM) during the pre-task period, increased to $84.0 \pm 8.00$ BPM during the task period, and subsequently decreased to $79.6 \pm 6.29$ BPM. A two-way ANOVA with group and period as independent variables was used to examine heart rate responses. The ANOVA revealed a significant main effect of period $(\mathrm{F}(2,30)=5.91, p<.01)$. The subsequent Tukey's honestly significant difference (HSD) test comparisons indicated that heart rate significantly increased from the pre-task to the task period $(p<.05)$, and decreased significantly to the posttask period $(p<.05)$.

\section{Mood Responses during and after Mental Stress Testing}

Two two-way ANOVAs were used to test whether change patterns of mood responses would be different between the two groups. Figures $\mathbf{2}$ and $\mathbf{3}$ show changes of the energetic arousal scores. ANOVA for the energetic arousal score revealed sig-

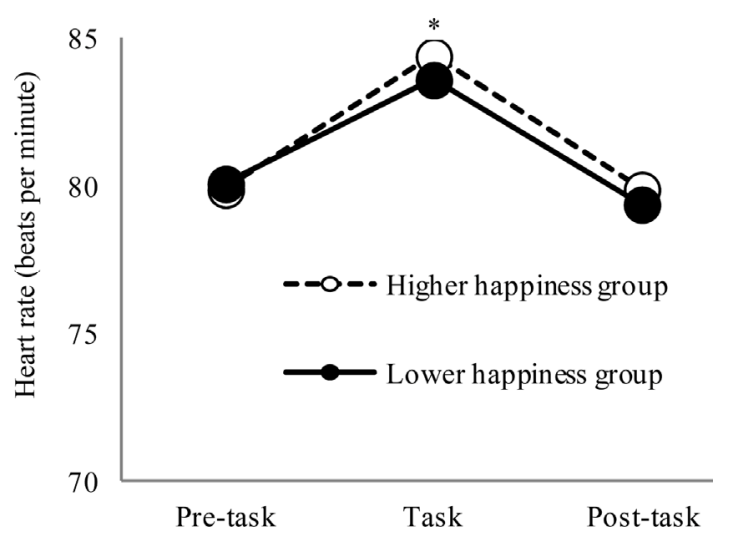

Figure 1.

Heart rate responses before, during, and after mental stress testing in higher (solid line) and lower happiness groups (dashed line). ${ }^{*} p<.05$ (vs. pre-task, post-task). 


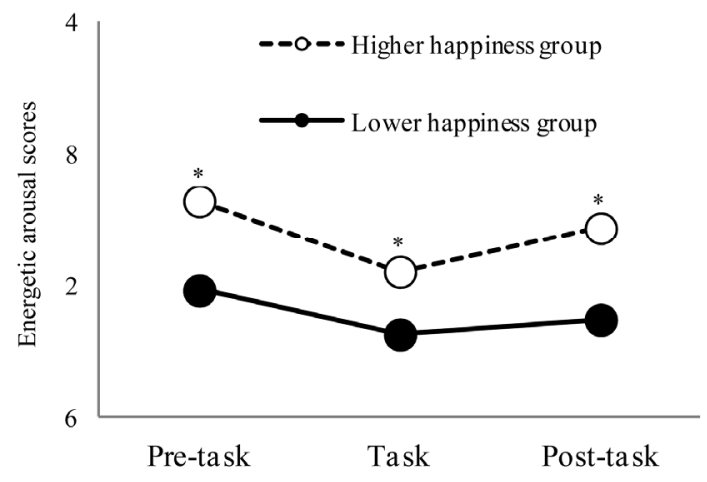

Figure 2.

Energetic arousal scores before, during, and after mental stress testing in higher (dashed line) and lower happiness groups (solid line). ${ }^{*} p<.01$ (vs. with lower happiness group).

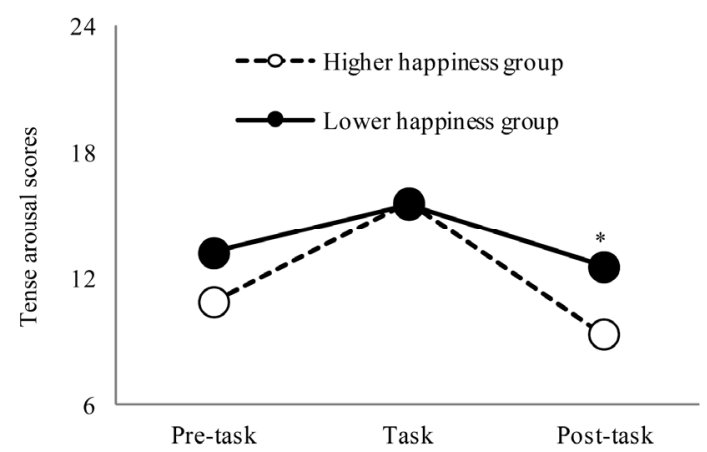

Figure 3.

Tense arousal scores before, during, and after mental stress testing in higher (dashed line) and lower happiness groups (solid line). ${ }^{*} p<.01$ (vs. with lower happiness group).

nificant main effects of group $(\mathrm{F}(1,15)=6.43, p<.05)$ and period $(\mathrm{F}(2,30)=8.23, p<.01)$. Interaction effect was not significant $(\mathrm{F}(2,30)=0.67$, n.s. $)$. The energetic arousal score was significantly higher in the HG than in LG. The subsequent Tukey's HSD comparisons indicated that the energetic arousal score was significantly higher in the pre-task compared to the task period $(p<.05)$.

ANOVA for the tense arousal score revealed significant effects of interaction $(\mathrm{F}(2,30)=3.58, p<.05)$ and period $(\mathrm{F}(2$, $30)=29.44, p<.01$.). Main effect of group was not significant $(\mathrm{F}(1,15)=1.63$, n.s. $)$. The subsequent analyses of the interaction effect indicated that the tense arousal score was significantly higher at the post-task period in LG than in HG. Also, in both groups, the score increased from the pre-task to the task period $(p<.05)$ and then decreased significantly during the post-task period $(p<.05)$.

\section{Discussion}

This study adopted a four-minute mental arithmetic task in front of an evaluative observer to induce stress. Heart rate increased during mental stress testing and returned to the baseline level four minutes after the test ended. These changes in heart rate were consistent with those of previous studies (Bostock et al., 2011; Brummett et al., 2009; Steptoe et al., 2007), which reported that mental stress testing results in an increased heart rate. No significant differences were found in objective per- formance between the two happiness groups; this was consistent with the results of Bostock et al. (2011) and Lyubomirsky and Ross (1997) that happiness was not related to objective performance. Therefore, the mental arithmetic task successfully induced a transiently stressed state.

Hypothesis 1 was not supported. No significant differences in negative and positive moods during mental stress testing were found. Our results were not consistent with those of Lyubomirsky and Ross (1997) and Lyubomirsky et al. (2001). One possibility for the inconsistency may be that the present study, unlike previous studies, did not provide negative social comparison information for each participant. For example, Lyubomirsky et al. (2001) found that unhappy students had significantly reduced positive moods and heightened negative moods when they received unfavorable news (i.e., their team lost a competition). No differences were found in mood responses related to favorable news (i.e., their team won). These results indicate that happiness moderates individual's mood responses when social comparison information is unfavorable. This study included an evaluative observer; however, direct unfavorable social comparison information was not provided. Rather, on an average, $80 \%$ of the feedback received by participants during the task period was positive. The differences in experimental procedures may account for the inconsistent results.

In contrast, hypothesis 2 was partially supported. Negative mood levels were lower in happier students following mental stress testing, but differences were not found in relation to positive moods. Significant relationships between happiness and the post-task negative moods were found, consistent with the results of Papousek et al. (2010), and inconsistent with those of Bostock et al. (2011). Differential changes in tense and energetic arousals from the task to the post-task period were observed. The changes in the scores of energetic arousal were compatible in both groups, while those in tense arousal differed. Tense and energetic arousals have been considered as two distinct types of activation (Schimmack \& Reisenzein, 2002) having differential correlates. For example, energetic arousal is related to performance more strongly than tense arousal (Matthews \& Davies, 2001; Matthews \& Westerman, 1994). Since objective performance was not significantly different between groups, discrepancies between changes in negative and positive mood changes from the task to the post-task period are understandable.

No effect was found in heart rate response. However, the changes in moods following mental stress testing do not necessarily correspond to cardiovascular responses. Waugh, Panage, Mendes, and Gotlib (2010) analyzed the correlation of heart rate and affective responses after giving a public speech, as well as following anticipation of giving a speech. They found significant correlations resulting only from anticipation. Discrepancies between heart rate and mood responses are acceptable.

The present results have made the following contributions to the literature: First, this study is one of the first to show that happiness, measured using SHS, is related to lower levels of tension and anxiety following mental stress testing. Papousek et al. (2010) related trait positive affect, measured using PANAS, to mood responses. Bostock et al. (2011) examined positive emotional style defined as the average level of a variety of experienced positive emotions over a seven-day period. When measured using SHS, happiness differs from these trait positive 
affect insofar as happiness in this study refers to one's global evaluation of being happy.

Second, this study examined Japanese students. The definition of happiness varies between western and eastern countries. Happiness is a self-related concept in western countries, while it is a social concept in eastern countries, such as Japan. High levels of happiness in North America, for example, are related to increased self-achievement and self-esteem, while happiness in Japan tends to be related to social relationships and harmony. This study's results suggest the possibility that happiness buffers the impacts of stress for Japanese individuals by reducing the negative mood following a stressful situation. These effects are vital for practical purposes. Although the importance of increasing positive affect and happiness in stress management is well documented (Cabinet Office of Japan, 2008), there is no evidence in Japan regarding the stress-buffering properties of happiness. This study represents some of the first findings to suggest the presence of stress-buffering properties, and to provide further understanding for Japanese practitioners who may incorporate happiness-enhancing intervention methods in stress management programs.

In conclusion, this study's results suggest that happiness buffers the impact of stressors on negative moods by influencing post-stressor negative mood levels in Japanese college students. Although this study is novel to Japan, several limitations must be addressed in future studies. First, only heart rate and mood responses were examined in the study. Previous studies reported that happiness and positive affect are related to quicker stabilization of blood pressure (Bostock et al., 2011; Steptoe et al., 2007). Therefore, it is necessary to assess other cardiovascular measures. Second, this study examined only two happiness groups. It is necessary to also include a group of individuals with average levels of happiness. Such an examination will increase our understanding of the ways in which happiness influences mood responses both during and after mental stress testing.

\section{Acknowledgements}

This work was supported by Grant-in-Aid for Scientific Research (A) (18203035) and (B) (22330196) to A. T. and a grant-in-Aid for JSPS fellows (12J07469) to S. H. We thank Dr. Yuichiro Nagano for providing us with the mental arithmetic task and Mr. Kengo Mihara for his help in preparing the manuscript. This paper is a part of the modified version of N. Toyoshima's master's dissertation.

\section{REFERENCES}

Anderson, N. B., Nordal, K. C., Breckler, S. J., Ballard, D., Bufka, L., Bossolo, L. et al. (2010). Stress in America findings. American Psychological Association.

http://www.apa.org/news/press/releases/stress/national-report.pdf

Bostock, S., Hamer, M., Wawrzyniak, A. J., Mitchell, E. S., \& Steptoe, A. (2011). Positive emotional style and subjective, cardiovascular and cortisol responses to acute laboratory stress. Psychoneuroendocrinology, 36, 1175-1183. doi:10.1016/j.psyneuen.2011.02.009

Brosschot, J. F., Gerin, W., \& Thayer, J. F. (2006). The perseverative cognition hypothesis: A review of worry, prolonged stress-related physiological activation, and health. Journal of Psychosomatic Research, 60, 113-124. doi:10.1016/j.jpsychores.2005.06.074

Brummett, B. H., Boyle, S. H., Kuhn, C. M., Siegler, I. C., \& Williams, R. B. (2009). Positive affect is associated with cardiovascular reactivity, norepinephrine level, and morning rise in salivary cortisol.
Psychophysiology, 46, 862-869.

doi:10.1111/j.1469-8986.2009.00829.x

Cabinet Office of Japan (2008). White paper on the national lifestyle 2008: Prospects for consumer citizenship.

http://www5.cao.go.jp/seikatsu/whitepaper/h20/06_eng/chapter1-3.p df

Campbell, A. (1981). The sense of well-being in America: Recent patterns and trends. New York: Mcgraw-Hill.

Emmons, R. A. (1986). Personal strivings: An approach to personality and subjective well being. Journal of Personality and Social Psychology, 51, 1058-1068. doi:10.1037/0022-3514.51.5.1058

Greenberg, J. S. (2010). Comprehensive stress management (12th ed.). New York: McGraw-Hill Companies.

Horiuchi, S., Tsuda, A., Hashimoto, E., Kai, H., \& Wenjie, H. (2008). Effect of perceived happiness level on cardiac response to mental stress testing: A pilot study. Japanese Journal of Biofeedback Research, 35, 93-98.

Horiuchi, S., Tsuda, A., Okamura, H., Yajima, J., \& Steptoe, A. (2010). Differential elicitation of the salivary 3-Methoxy-4-hydro xyphenylglycol (MHPG) responses by mental stress testing. Japanese Journal of Behavioral Medicine, 16, 31-38.

Kwan, V. S. Y., Bond, M. H., \& Singelis, T. S. (1997). Pancultural explanations for life satisfaction: Adding relationship harmony to self-esteem. Journal of Personality and Social Psychology, 73, 10381051. doi: $10.1037 / 0022-3514.73 .5 .1038$

Lazarus, R. S. (2009). Stress and emotion: A new synthesis. New York: Springer.

Lazarus, R. S., \& Folkman, S. (1984). Stress, appraisal, and coping. New York: Springer.

Lyubomirsky, S. (2000). Why are some people happier than others? The role of cognitive and motivational processes in well-being. American Psychologist, 56, 239-249.

doi:10.1037/0003-066X.56.3.239

Lyubomirsky, S., \& Lepper, H. S. (1999). A measure of subjective happiness: Preliminary reliability and construct validation. Social Indicators Research, 46, 137-155. doi:10.1023/A:1006824100041

Lyubomirsky, S., \& Ross, L. (1997). Hedonic consequences of social comparison: A contrast of happy and unhappy people. Journal of Personality and Social Psychology, 73, 1147-1157. doi:10.1037/0022-3514.73.6.1141

Lyubomirsky, S., Tkach, C., \& DiMatteo, M. R. (2006). What are the differences between happiness and self-esteem? Social Indicators Research, 78, 363-404. doi:10.1007/s11205-005-0213-y

Lyubomirsky, S., Tucker, K. L., \& Kasri, F. (2001). Responses to hedonically conflicting social comparison: Comparing happy and unhappy people. European Journal of Social Psychology, 31, 511-535. doi:10.1002/ejsp.82

Matsunaga, M., Murakami, H., Yamakawa, K., Isowa, T., Fukuyama, S., Shinoda, J. et al. (2011). Perceived happiness level influences evocation of positive emotions. Natural Science, 3, 723-727. doi:10.4236/ns.2011.38095

Matthews, G., \& Davies, D. R. (2001). Individual differences in energetic arousal and sustained attention: A dual-task study. Personality and Individual Differences, 31, 575-589. doi:10.1016/S0191-8869(00)00162-8

Matthews, G., \& Westerman, S. J. (1994). Energy and tension as predictors of controlled visual and memory search. Personality and Individual Differences, 17, 617-626. doi:10.1016/0191-8869(94)90134-1

McEwen, B. S. (1998). Stress, adaptation, and disease: Allostasis and allostatic load. Annals of the New York Academy of Sciences, 840, 33-44. doi:10.1111/j.1749-6632.1998.tb09546.x

Nelson, D., \& Cooper, C. (2005). Stress and health: A positive direction. Stress and Health, 21, 73-75. doi:10.1002/smi.1053

Okamura, H., Tsuda, A., \& Yajima, J. (2004). Stress state questionnaire. In Public Research Center (Eds.), Stress scale guidebook (pp. 214220). Tokyo: Jitsumukyoiku-Shuppan.

Papousek, I., Nauschnegg, K., Paechter, M., Lackner, H. K., Goswami, N., \& Schulter, G. (2010). Trait and state positive affect and cardiovascular recovery from experimental academic stress. Biological Psychology, 83, 108-115. doi:10.1016/j.biopsycho.2009.11.008 
Schiffrin, H. H., \& Nelson, S. K. (2010). Stressed and happy? Investigating the relationship between happiness and perceived stress. Journal of Happiness Studies, 11, 33-39.

doi:10.1007/s10902-008-9104-7

Schimmack, U., \& Reisenzein, R. (2002). Experiencing activation: Energetic arousal and tense arousal are not mixtures of valence and activation. Emotion, 2, 412-417. doi:10.1037/1528-3542.2.4.412

Shimai, S., Otake, K., Utsuki, N., Ikemi, A., \& Lyubomirsky, S. (2004). Development of a Japanese version of the subjective happiness scale (SHS), and examination of its validity and reliability. Japanese Journal of Public Health, 51, 845-853.

Stawski, R. S., Sliwinski, M. J., Almeida, D. M., \& Smyth, J. M. (2008). Reported exposure and emotional reactivity to daily stressors: The roles of adult age and global perceived stress. Psychology and Aging, 23, 52-61. doi:10.1037/0882-7974.23.1.52

Steptoe, A., Gibson, E. L., Hamer, M., \& Wardle, J. (2007). Neuroendocrine and cardiovascular correlates of positive affect measured by ecological momentary assessment and by questionnaire. Psychoneuroendocrinology, 32, 56-64. doi:10.1016/j.psyneuen.2006.10.001
Uchida, Y., \& Kitayama, S. (2009). Happiness and unhappiness in east and west: Themes and variations. Emotion, 9, 441-456. doi: $10.1037 / \mathrm{a} 0015634$

Uchida, Y., Kitayama, S., Mesquita, B., Reyes, J. A., \& Morling, B. (2008). Is perceived emotional support beneficial? Well-being and health in independent and interdependent cultures. Personality and Social Psychology Bulletin, 34, 741-754. doi: $10.1177 / 0146167208315157$

Watson, D., Clark, L. A., \& Tellegen, A. (1988). Development and validation of brief measures of positive and negative affect: The PANAS scales. Journal of Personality and Social Psychology, 54, 1063-1070. doi:10.1037/0022-3514.54.6.1063

Watts, J., \& Robertson, N. (2011). Burnout in university teaching staff: A systematic literature review. Educational Research, 53, 33-50. doi:10.1080/00131881.2011.552235

Waugh, C. E., Panage, S., Mendes, W. B., \& Gotlib, I. H. (2010). Cardiovascular and affective recovery from anticipatory threat. Biological Psychology, 84, 169-175. doi:10.1016/j.biopsycho.2010.01.010 